\title{
POLOCAM: a millimeter wavelength cryogenic polarimeter prototype for MUSIC-POL
}

\author{
Glenn T. Laurent ${ }^{a}$, John E. Vaillancourt ${ }^{b}$, Giorgio Savini $^{c}$, Peter A.R. Ade ${ }^{d}$, Stephane \\ Beland $^{e}$, Jason Glenn ${ }^{f}$, Matthew I. Hollister ${ }^{g}$, Philip R. Maloney ${ }^{f}$ and Jack Sayers ${ }^{h}$ \\ ${ }^{a}$ Southwest Research Institute, 1050 Walnut St. Suite 300, Boulder, CO 80302, USA; \\ ${ }^{b}$ SOFIA Science Center, USRA, NASA Ames Research Center, MS 211-3, Moffett Field, CA \\ 94035, USA; \\ ${ }^{c}$ Department of Physics and Astronomy, University College London, Gower Street, London, \\ WC1E 6BT, UK; \\ ${ }^{d}$ School of Physics and Astronomy, Cardiff University, The Parade, Cardiff, CF24 3AA, UK; \\ ${ }^{e}$ LASP, University of Colorado, 1234 Innovation Dr., Boulder, CO 80303, USA; \\ ${ }^{f}$ CASA, University of Colorado, 389 UCB, Boulder, CO 80309, USA; \\ ${ }^{g}$ Jet Propulsion Laboratory, Pasadena, CA 91109, USA; \\ ${ }^{h}$ Caltech, MC367-17, Pasadena, CA 91125, USA
}

\begin{abstract}
As a proof-of-concept, we have constructed and tested a cryogenic polarimeter in the laboratory as a prototype for the MUSIC instrument (Multiwavelength Sub/millimeter Kinetic Inductance Camera). The POLOCAM instrument consists of a rotating cryogenic polarization modulator (sapphire half-waveplate) and polarization analyzer (lithographed copper polarizers deposited on a thin film) placed into the optical path at the Lyot stop (4 K cold pupil stop) in a cryogenic dewar. We present an overview of the project, design and performance results of the POLOCAM instrument (including polarization efficiencies and instrumental polarization), as well as future application to the MUSIC-POL instrument.
\end{abstract}

Keywords: polarimetry, MUSIC, submillimeter detectors, MKID, cryogenics

\section{INTRODUCTION}

Magnetic fields remain one of the least understood components of the interstellar medium (ISM). Permeating the ISM on essentially all scales, they may play a vital role in the large-scale vertical structure of the ISM in galactic disks, ${ }^{1}$ and significantly influence the gravitational collapse of molecular clouds to form both highmass and low-mass stars. ${ }^{2-5}$ Measurements of the polarized radiation from interstellar dust grains which are aligned with magnetic fields allow one to map the orientation of the field projected onto the plane of the sky. ${ }^{6-8}$ However, existing observations are limited to the brightest clouds ${ }^{9-11}$ or rely on large beam sizes to enhance sensitivity while compromising spatial resolution. ${ }^{12-15}$ These limitations have hampered attempts to use polarization measurements to quantify the role magnetic fields play in the dynamics of the ISM.

By taking advantage of recent developments in detector technology, it is possible to derive information on magnetic fields in Galactic molecular clouds through mapping of polarized thermal dust emission with high speed and sensitivity. MUSIC (the Multiwavelength Submillimeter kinetic Inductance Camera) ${ }^{16}$ is an instrument recently built for ground-based astronomical observations at (sub)millimeter wavelengths from the Caltech Submillimeter Observatory (CSO) which utilizes a new type of superconducting detector that allows simultaneous four-color, multi-wavelength observations. The heart of MUSIC is an array of Microwave Kinetic Inductance Detectors (MKIDs), which make use of the change in surface impedance of a superconductor as incoming photons break Cooper pairs. ${ }^{17}$ An advantage of this technology is the use of frequency-domain multiplexing which allows

Further author information: (Send correspondence to Glenn T. Laurent)

E-mail: glaurent@boulder.swri.edu, Telephone: 17202400155

Millimeter, Submillimeter, and Far-Infrared Detectors and Instrumentation for Astronomy VI, edited by Wayne S. Holland, Jonas Zmuidzinas, Proc. of SPIE Vol. 8452,

84521S @ 2012 SPIE · CCC code: 0277-786X/12/\$18 · doi: 10.1117/12.926430

Proc. of SPIE Vol. 845284521 S-1 
hundreds of resonators (tuned to slightly different frequencies) to be simultaneously read-out through a single coaxial transmission line. Future plans for the MUSIC instrument include the construction and operation of a cryogenically cooled polarimeter (MUSIC-POL) that will be used in conjunction with MUSIC at the CSO to map the polarized emission from Galactic molecular clouds at wavelengths of $0.85-2 \mathrm{~mm}$. With a 576 pixel array, the MUSIC camera promises a mapping speed advantage of a factor of 6 (at $1.1 \mathrm{~mm}$ ) over the instrument it is replacing (BOLOCAM). Furthermore, as the MUSIC instrument is inherently a single polarization instrument, no significant additional observing efficiency loss (beyond the need for measurements at multiple waveplate angles) is expected when adding the polarization attachment. In addition to the dramatic improvement in observing efficiency, simultaneously imaging all four colors with the MUSIC polarimeter (MUSIC-POL) provides the unique advantage of minimizing systematic uncertainties between the passbands (from varying instrument efficiencies, calibrations, etc...). One of the primary science drivers that has motivated the MUSIC project is to reveal the processes of formation and evolution of molecular clouds, from the interstellar medium to star formation. ${ }^{18}$ As polarized emission from Galactic molecular clouds trace the magnetic fields that permeate them, the development of the MUSIC-POL instrument will help contribute to these goals.

\section{POLOCAM DESIGN}

The POLOCAM instrument was designed as a prototype for the MUSIC-POL instrument with the primary goal of constructing a working cryogenic polarimeter in the laboratory. An existing warm polarimeter (built and tested on the BOLOCAM instrument, which MUSIC is superseding) could potentially be installed on MUSIC without a lengthy cryostat warm-up and cool-down period. However, a cold waveplate system addresses two important systematic limitations of these room-temperature polarimeters. First, MUSIC-POL is designed such that the waveplate is located at the pupil stop. In this way, all of the beams will illuminate the waveplate as uniformly as possible, and will do so for all rotation angles. As a result, any systematic polarization that might be introduced due to waveplate misalignment, flaws, or vignetting will be minimized.

Second, the optical loading from a cold waveplate (at MUSIC's $4 \mathrm{~K}$ Lyot stop) will be significantly less than that for a warm waveplate (or analyzer). At $1 \mathrm{~mm}$, room-temperature crystal sapphire has ordinary and extraordinary indicies of refraction of $n_{o}=3.068$ and $n_{e}=3.409$, with corresponding absorption coefficients of $\alpha_{o} \simeq 0.01$ and $\alpha_{e} \simeq 0.04 \mathrm{~cm}^{-1}$, respectively. At $4 \mathrm{~K}$, the indicies of refraction and absorption coefficients become $n_{o}=3.050, n_{e}=3.362$ and $\alpha_{o} \simeq 0.001$ and $\alpha_{e} \simeq 0.0025 \mathrm{~cm}^{-1}$, respectively. These indicate first-order half-waveplate thicknesses of 1.6 and $1.7 \mathrm{~mm}$ at $300 \mathrm{~K}$ and $4 \mathrm{~K}$, respectively. For warm and cold waveplates, the polarization induced by differential reflection and absorption is approximately $\lesssim 1 \%$ and $\lesssim 0.1 \%$, respectively, taking into account the reductions from the anti-reflection coatings. However, accounting for the instrumental transmission, the in-band polarized flux from the waveplate at the detectors is $\sim 12 \mathrm{pW}$ for the room-temperature waveplate, and $\sim 0.9 \mathrm{pW}$ for the $4 \mathrm{~K}$ waveplate, compared to an expected (unpolarized) load from the telescope and sky of $\sim 10 \mathrm{pW}$. The polarized flux from the $4 \mathrm{~K}$ waveplate will be both lower and more stable since it will be inside the innermost layer of the cryostat (with a constant temperature), making it easier to subtract than the polarized flux from a warm waveplate. Thus, while a rotating a cryogenic waveplate is more complex than a room-temperature implementation and requires a warm-up and cool-down period between observing runs (for installation), the induced systematic polarization will be reduced considerably and justifies the additional effort.

As a proof-of-concept, we have constructed and tested a cryogenic polarimeter in the laboratory. The POLOCAM instrument consists of a cryogenic polarization modulator (sapphire half-waveplate) and polarization analyzer (lithographed copper polarizers deposited on a thin film) placed into the optical path at the Lyot stop ( $4 \mathrm{~K}$ cold pupil stop) in a cryogenic dewar. The waveplate is mounted on a large diameter turntable bearing designed to rotate at cryogenic temperatures and is coupled to a warm stepper motor outside the dewar via a flexible coupling and a vacuum-tight ferro-fluidic feedthrough.

Figure 1 shows the design of the rotating POLOCAM waveplate assembly. The primary optical component of POLOCAM consists of a half wave plate (HWP) that is designed to modulate incoming polarized emission and enable measurement of the three Stokes parameters describing linear polarization $(I, Q, U)$. The waveplate itself consists of five chemically polished, X-cut crystal sapphire slabs which provide orthogonal fast and slow electromagnetic wave propagation axes. High precision sapphire waveplates (birefringent) were fabricated in April 2009 by Crystran Ltd. and delivered to Cardiff University for multi-layered anti-reflection coating and 


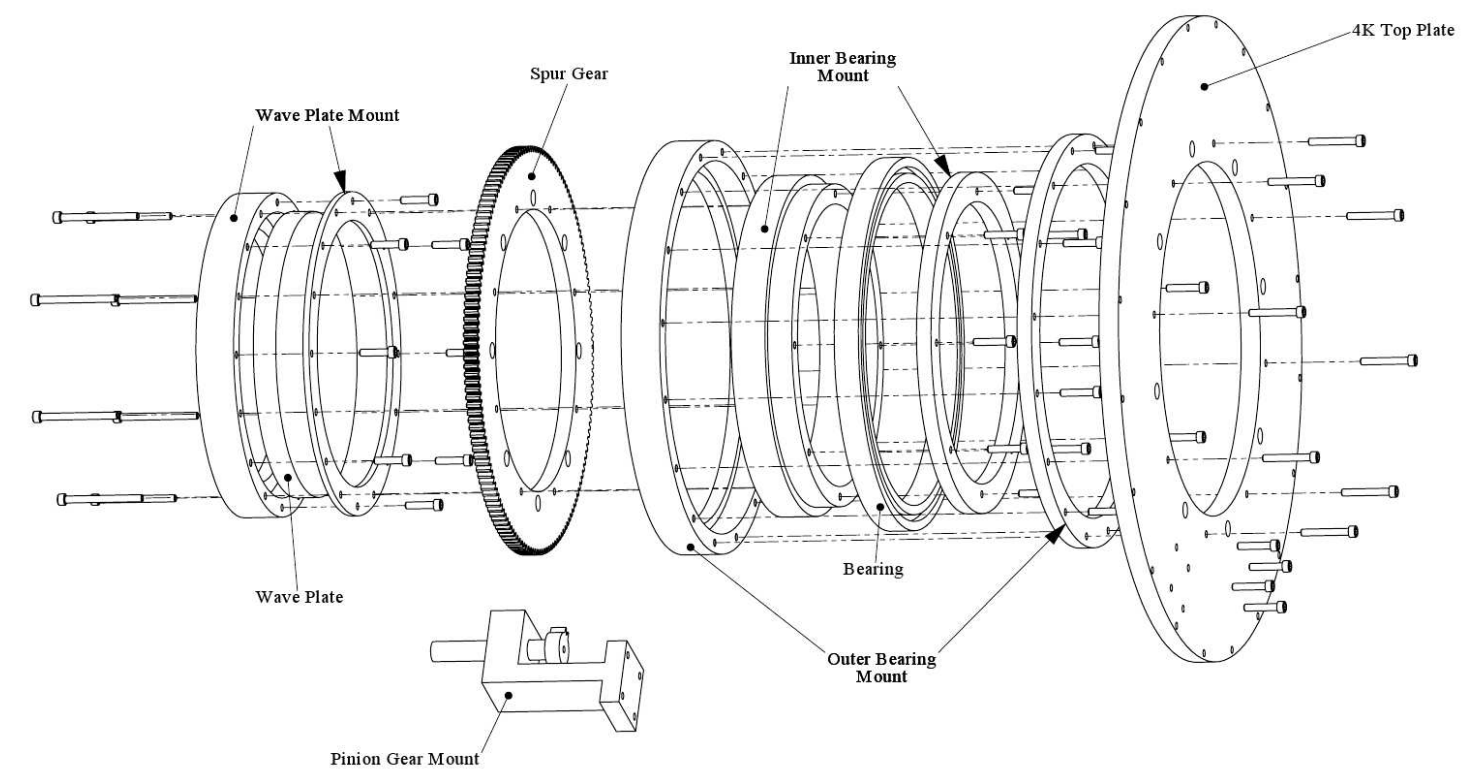

Figure 1. POLOCAM rotating waveplate assembly design.

bonding. The anti-reflection coating was constructed by photolithographically depositing patterns of copper on thin substrates of polypropylene, assembling multiple layers into a stack with an additional outer layer of porous PTFE, and finally fusing the multi-layered structures onto each side of the HWP by hot pressing at a temperature near the polypropylene melting point. ${ }^{19}$ The individual half-waveplates were stacked with their fast axes of polarization offset by \pm 60 degrees such that the combined waveplate has a fast-action direction that is only mildly wavelength dependent with a high polarization modulation efficiency across the entire band. The waveplate and antireflection coating thicknesses were designed for achromatic performance across the preliminary MUSIC wavebands $(230,285,345$, and $408 \mathrm{GHz})$, with high fabrication tolerances to minimize instrumental polarization due to non-parallel surfaces. The resulting waveplate was repeatedly thermally cycled to $4 \mathrm{~K}$ to ensure proper AR-coating lamination. The HWP was then unit-level tested at room temperature for transmission and frequency behavior using a Martin-Puplett type polarizing Fourier Transform Spectrometer (FTS) with an incoherent mercury arc lamp source at Cardiff University. The waveplate was positioned between two $10 \mu \mathrm{m}$ period photo-lithographic grid polarizers and tested in a high-accuracy $(\leq 0.01 \mathrm{deg})$ rotating mount over a wide frequency range $(90-1100 \mathrm{GHz})$ using a $\mathrm{He} 3$ cooled $(380 \mathrm{mK})$ bolometer. The HWP was characterized in four configurations, with the waveplate fast axis oriented at $0,45,90$, and $135^{\circ}$ relative to the polarizing grids. The $0^{\circ}$ and $90^{\circ}$ measurements provide the transmission along the principle axes of the waveplate, while the $45^{\circ}$ and $135^{\circ}$ angles measure the cross-polarization. Figure 2 shows the resulting transmission efficiency $(\sim 70-90 \%)$ over the optimized frequency range, with a small cross-polarization leak of the waveplate itself $(\sim 0-2 \%)$ as tested with the FTS.

Figure 3 shows the final POLOCAM assembly, including the waveplate, bearings, spur and pinion gears, and cryogenic encoder. The waveplate is mounted to the inner race of a rotating bearing that has been specially designed to freely rotate at cryogenic temperatures. Specifically, the race clearances and bearing spacers were designed to eliminate binding due to thermal contraction. The final custom bearing (REALI-SLIM, Kaydon Inc.) was verified to rotate freely at $4 \mathrm{~K}$ in the POLOCAM test cryostat (see Section 3 ). The waveplate is driven by a combination spur/pinion gear. As shown in Figures 1 and 3, the spur gear and waveplate assembly are mounted to the inner race of the rotating bearing, and are driven by a smaller (10:1 gear ratio) pinion gear, which is mounted to the $4 \mathrm{~K}$ top plate of the POLOCAM cryostat. (The pinion gear is press-fit into a second, smaller bearing to ensure free rotation). A cryogenic encoder which directly measures the rotation angle of the waveplate at $4 \mathrm{~K}$ to within $\sim 0.4^{\circ}$ has been implemented (Figure 3 ). The cryogenic encoder utilizes three thin 


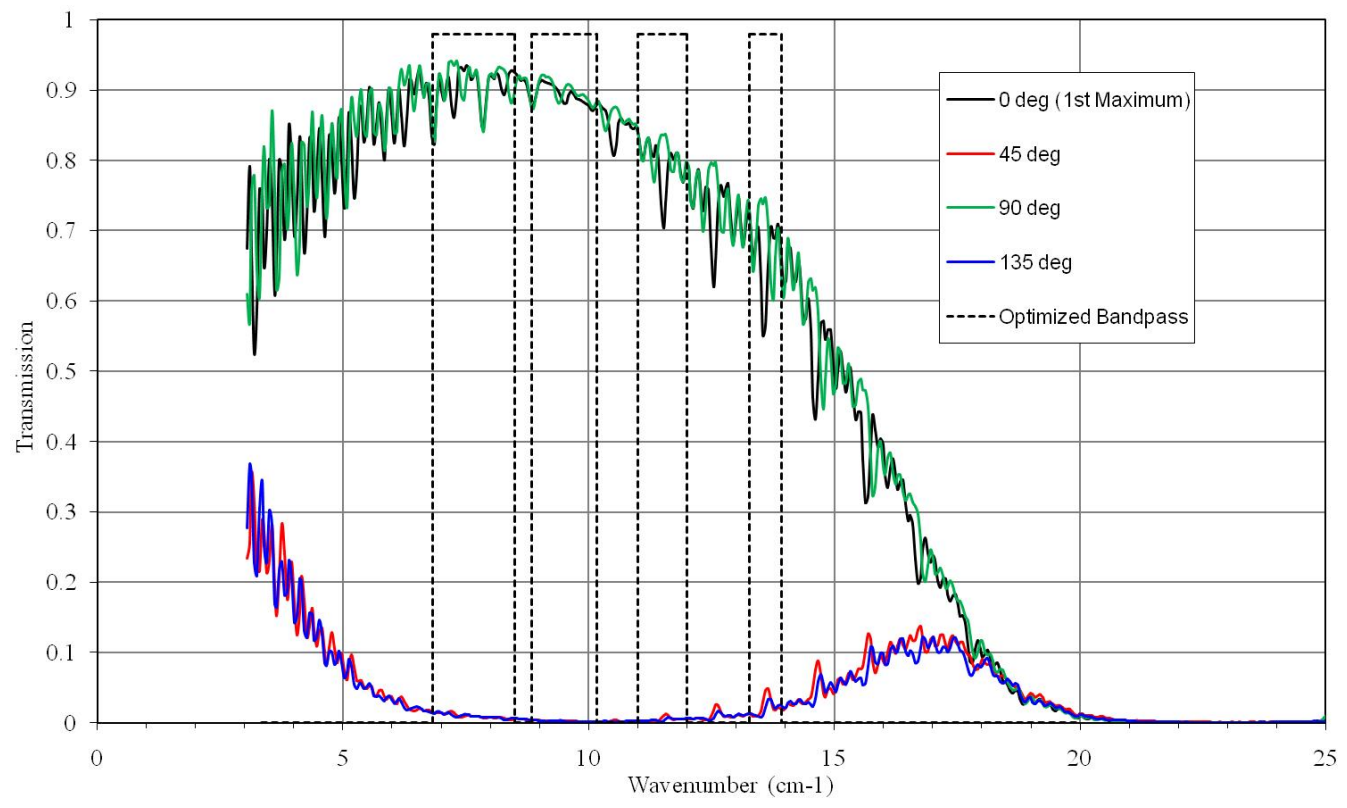

Figure 2. Unit-level FTS measurements of POLOCAM waveplate optical efficiencies. The black and green curves represent the polarization efficiency at waveplate angles of $0^{\circ}$ and $90^{\circ}$, respectively. The red and blue curves represent the crosspolarization leak at waveplate angles of $45^{\circ}$ and $135^{\circ}$, respectively. The angles are defined by the HWP fast axis relative to the two parallel polarizing grids. Dashed line: Preliminary MUSIC bandpasses.

wires mounted on the outer diameter of the rotating waveplate. Fixed-position brushes make contact with the wires, with the system acting as a voltage divider with warm low-noise preamplifier electronics. The calibration curve of the cryogenic encoder is repeatable and well behaved over multiple scans. The waveplate is designed to be rotated 22.5 degrees between subscans, enabling the Stokes parameters $Q$ and $U$ to be measured in four subscans.

The gear assembly is driven by a precision DC stepper motor and encoder assembly mounted external to the cryostat. Figure 5 shows the final design of the warm motor assembly. The motor is coupled to the pinion gear via a stock flexible coupling (McMaster Carr), which allows a horizontal rotating motor shaft (mounted on the side of the dewar) to turn the waveplate assembly about the vertical axis. As shown in Figure 5, at the insertion point of the dewar, a ferro-fluidic feedthrough (Ferrotec Inc.) allows mechanical coupling between the motor and the gears while ensuring against vacuum leaks. The thermal loading due to the warm G10 shaft penetrating the $4 \mathrm{~K}$ and $77 \mathrm{~K}$ vacuum shields is small with negligible effects on cryostat hold times and waveplate temperatures. An additional beam-style flexible coupler separates the stepper motor from the ferro-fluidic feedthrough to minimize vibrations (microphonics) internal to the dewar. An additional motor encoder was added as a redundant backup to the cryogenic encoder and allows for the direct readout of the motor position to within $\lesssim 0.1^{\circ}$ on the waveplate $\left(40,000\right.$ steps per $360^{\circ}$ waveplate rotation).

\section{POLOCAM TEST CRYOSTAT}

A cryogenic test dewar at the University of Colorado submillimeter laboratory was developed for the testing of the POLOCAM cryogenic waveplate. Figure 4 shows internal views of the completed dewar assembly. An existing IR Labs cryostat was available and was modified for use by both the POLOCAM team as well as being used for testing of optical components for the MUSIC project. Two views of the cryostat are shown in Figure 4: The left panel shows the $4 \mathrm{~K}$ radiation shield and machined window (with polypropylene filter on top) to which the POLOCAM waveplate assembly is to be mounted. G10 supports thermally isolate the $4 \mathrm{~K}, 77 \mathrm{~K}$, and $300 \mathrm{~K}$ stages; the right panel shows the gold plated $4 \mathrm{~K}$ stage and Chase Research $3 \mathrm{He} / 4 \mathrm{He}$ two-stage sorption fridge. A single bolometer and feedhorn (optimized for $850 \mu \mathrm{m}$ ) is mounted in the center of the image to the 

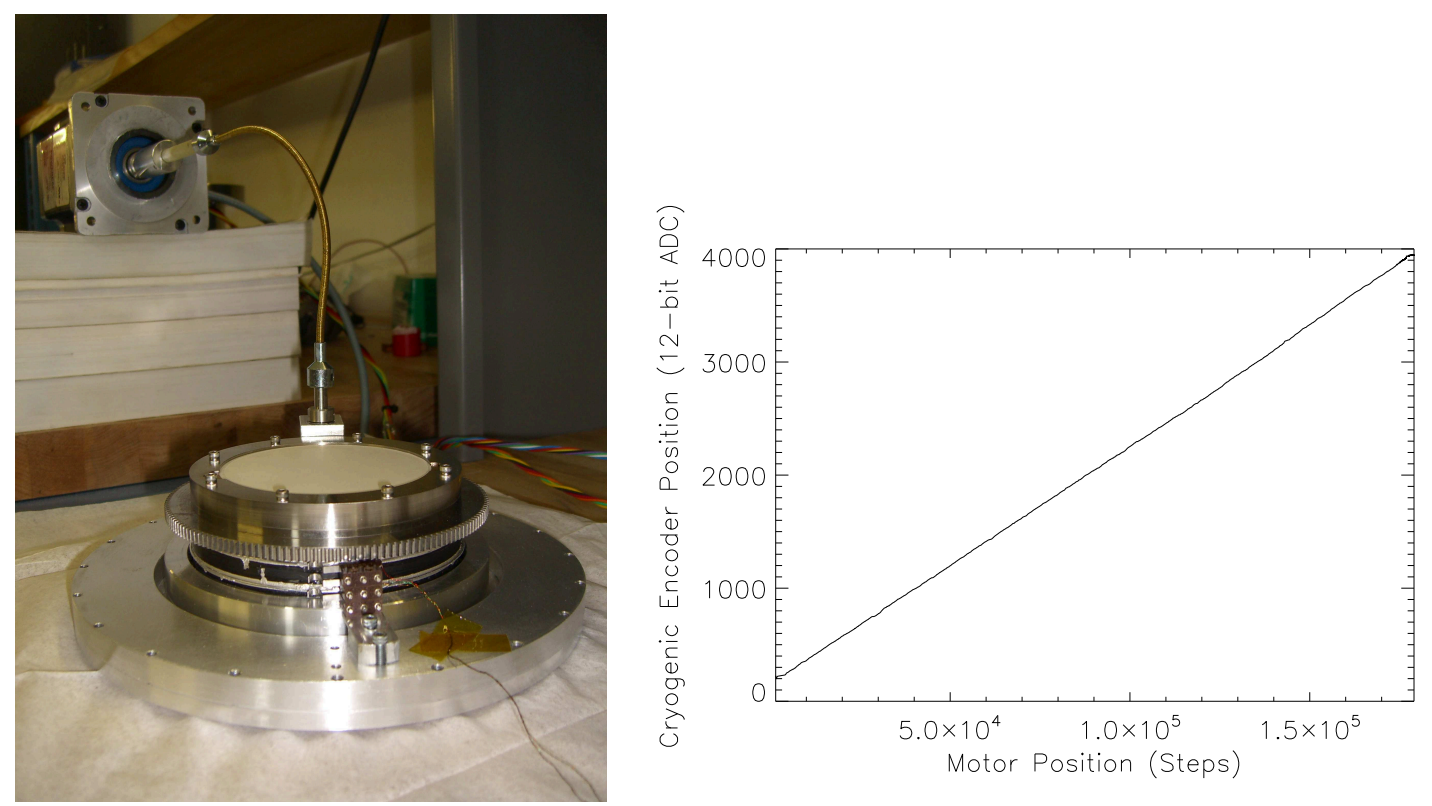

Figure 3. Left: Completed POLOCAM rotating waveplate and motor/encoder assembly. Right: $4 \mathrm{~K}$ calibration results of the cryogenic POLOCAM encoder over a single waveplate rotation.

ultracold stage. GRT resistance thermometry was been wired to the ultracold stage, with diodes on the $4 \mathrm{~K}$ and $77 \mathrm{~K}$ cold stages, waveplate mount, fridge stages, and JFETS, which allow for full monitoring of the cryostat and POLOCAM temperatures.
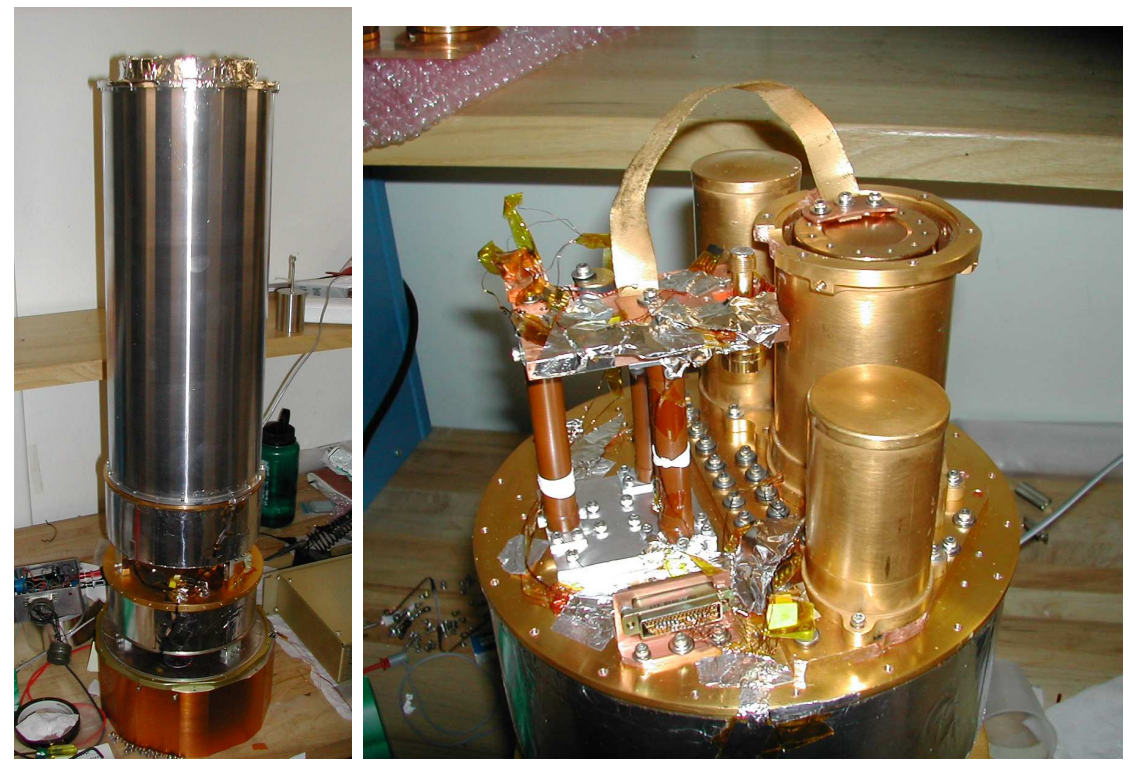

Figure 4. Left: Cryostat with $4 \mathrm{~K}$ POLOCAM radiation shield. Right: Interior view of the cryostat, showing the $4 \mathrm{~K}$ stage, fridge, ultracold stage, and bolometer/feedhorn assembly.

Thermal and mechanical testing of the cryostat was carried out at the submillimeter laboratory at the University of Colorado and show the $3 \mathrm{He} / 4 \mathrm{He}$ sorption fridge, GRT and diode thermometry to be working as anticipated. Cool-downs achieve stable $\mathrm{T} \sim 380 \mathrm{mK}$ temperatures at the ultracold (UC) stage, with the waveplate achieving a temperature of $10 \mathrm{~K}$. Commercially available digital electronics were used for the control and readout of the sorption fridge, bolometer, thermometry, and POLOCAM waveplate assembly. The electronics include DC 
power supplies for the cycling of the $3 \mathrm{He} / 4 \mathrm{He}$ fridge, a current source for DC biasing of the bolometer, a resistance bridge for the UC GRT readout, a custom breakout-box for intermediate measurements. To maximize the signalto-noise of the laboratory measurements, a hot blackbody source with a chopper-wheel was developed for testing, using a flat mirror to direct the source signal into the cryostat. A lock-in amplifier circuit was implemented to demodulate the chopped signal. End-to-end testing of all electrical component show all components to performing as designed. Figure 5 shows the completed POLOCAM test cryostat in a test configuration.
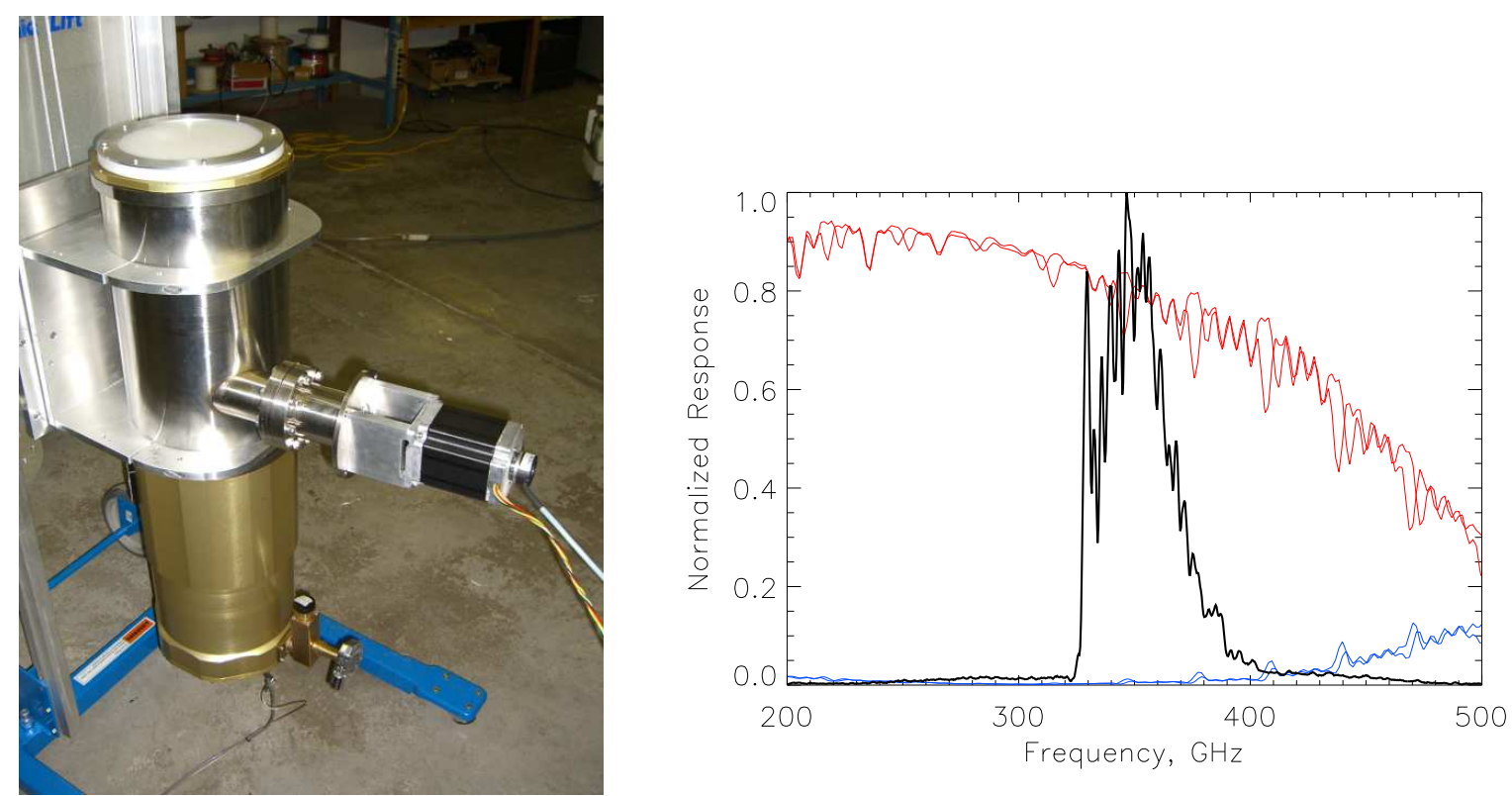

Figure 5. Left: Fully assembled POLOCAM test cryostat with warm motor and ferro-fluidic feedthrough assembly. Right: FTS frequency response of the POLOCAM cryostat (black). The polarization efficiencies (red) and cross-polarization leak (blue) data of unit-level testing of the waveplate from Figure 2 are also shown for reference.

Out-of-band radiation for the POLOCAM test cryostat is rejected with the use of polypropylene filters at the $300 \mathrm{~K}, 77 \mathrm{~K}$, and $4 \mathrm{~K}$ windows. The specific bandpass at the detector (optimized for $850 \mu \mathrm{m}$ ) is defined by the feedhorn/waveguide and metal-mesh filters (low-pass filters with cutoff frequencies of $360 \mathrm{and} 420 \mathrm{GHz}$ ) near the bolometer. Subsequent FTS testing of the cryostat shows the bandpass to be well defined and successful in suppressing out-of-band radiation (see Figure 5). Integrating the observed waveplate transmission efficiencies over the POLOCAM bandpass yields an expected overall polarization efficiency of $78 \%$ and cross-polarization leak of $0.8 \%$.

A thin, fixed-position membrane polarization analyzer was mounted to the $4 \mathrm{~K}$ Lyot stop, enabling the system to be sensitive to linear polarization. Another non-rotating analyzer (removable) was temporarily mounted on the exterior of the cryostat, to feed $100 \%$ polarized light to the waveplate in order to measure the instrumental polarization of the system. FTS testing show the polarizing grids to have high polarization efficiencies (99.099.5\% between $3-35 \mathrm{~cm}^{-1}$ ), with no distinguishable frequency dependence. Reduced optical efficiencies in initial testing were traced to the warm polarizing grid, which was originally oriented at normal incidence, thus reflecting the rejected aspect of polarization (from the cold analyzer) back into the cryostat. A 45-degree mount for the warm analyzer along with a shell lined with Eccosorb was developed to properly sink the rejected polarization signal outside the cryostat.

\section{OPTICAL EFFICIENCY MEASUREMENTS}

Assembly and end-to-end testing of the POLOCAM instrument was performed at the University of Colorado submillimeter laboratory. Results show the system to be functioning as designed mechanically and electrically. All components have been cryogenically tested for free rotation at $4 \mathrm{~K}$ in the POLOCAM cryostat. Instrument tests were performed in the laboratory, with the cryostat observing a chopped hot blackbody source. The system 
is driven with a custom developed LabView program, which steps the waveplate (using feedback from both the warm and cryogenic encoders) and integrates the DC-biased bolometer signal (after demodulating the chopped signal with a lock-in amplifier). Loadcurve measurements were performed to determine the optimal bias current for maximum bolometer responsivity.

POLOCAM response measurements were taken for both $100 \%$ polarized (using the warm polarizing analyzer) and unpolarized (no warm analyzer) source signals. In each case, the waveplate was stepped in $5^{\circ}$ increments and the signal integrated. Test results show the successful modulation of the waveplate with the expected $90^{\circ}$ period. Figure 6 shows the detector response to both polarized and unpolarized source signals while modulating the waveplate. Also shown in the figure is a sinusoidal fit (solid line) to the $90^{\circ}$ periodicity and zero-frequency offset terms. Repeated measurements show a small dispersion $(\sigma=0.4 \%)$, indicating proper electro-mechanical operation of the drive assembly and cryogenic encoder.

A Fourier transform analysis of these data separates the polarization signal with a periodicity of $90^{\circ}$ from other systematics and indicates a polarization efficiency of $80 \%$ and an instrumental polarization of 1.4\% (Figure 7). This is in good agreement with the expected behavior from Section 3. Additional instrumental polarization components with $180^{\circ}$ and $360^{\circ}$ periodicity are small $(0.3$ and $0.1 \%$, respectively) and are indicative of imperfections in the manufacturing process of the waveplate and mounting assembly (i.e. tilt).
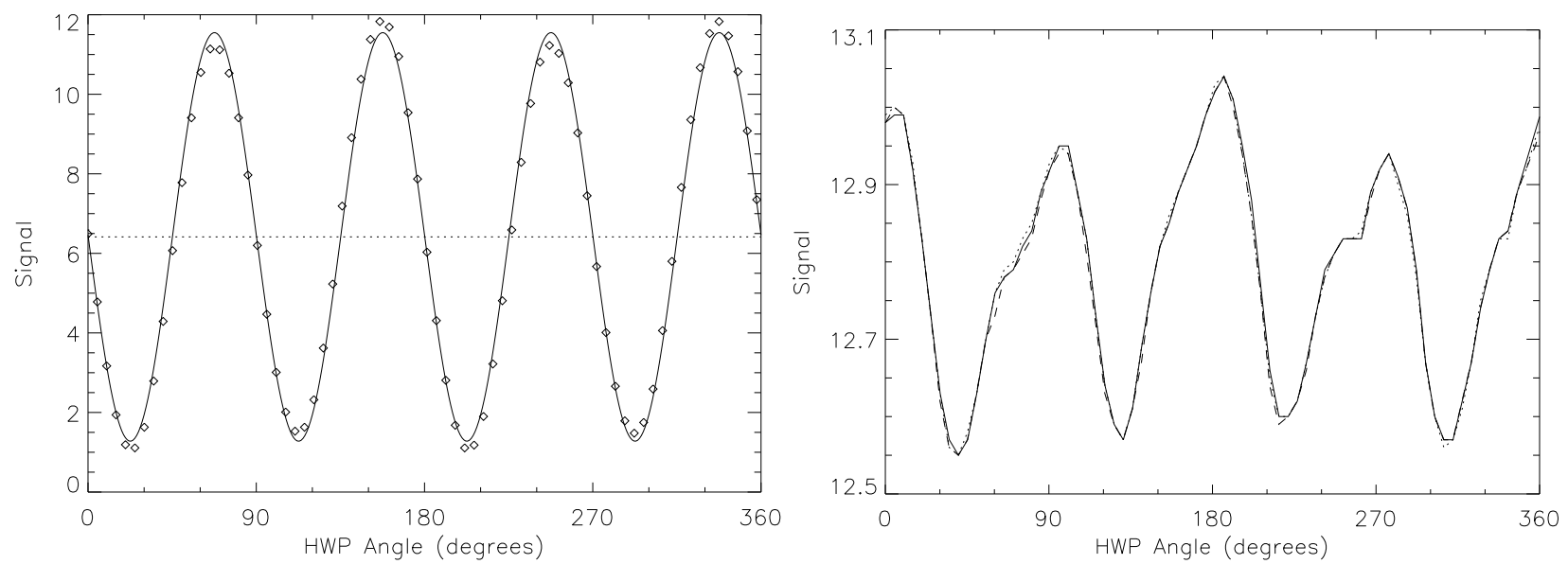

Figure 6. End-to-end POLOCAM response as a function of waveplate rotation angle for both $100 \%$ polarized (Left) and unpolarized (Right) source signals. Note the magnified scale in unpolarized source case, with a relatively small amplitude compared to the zero-offset.
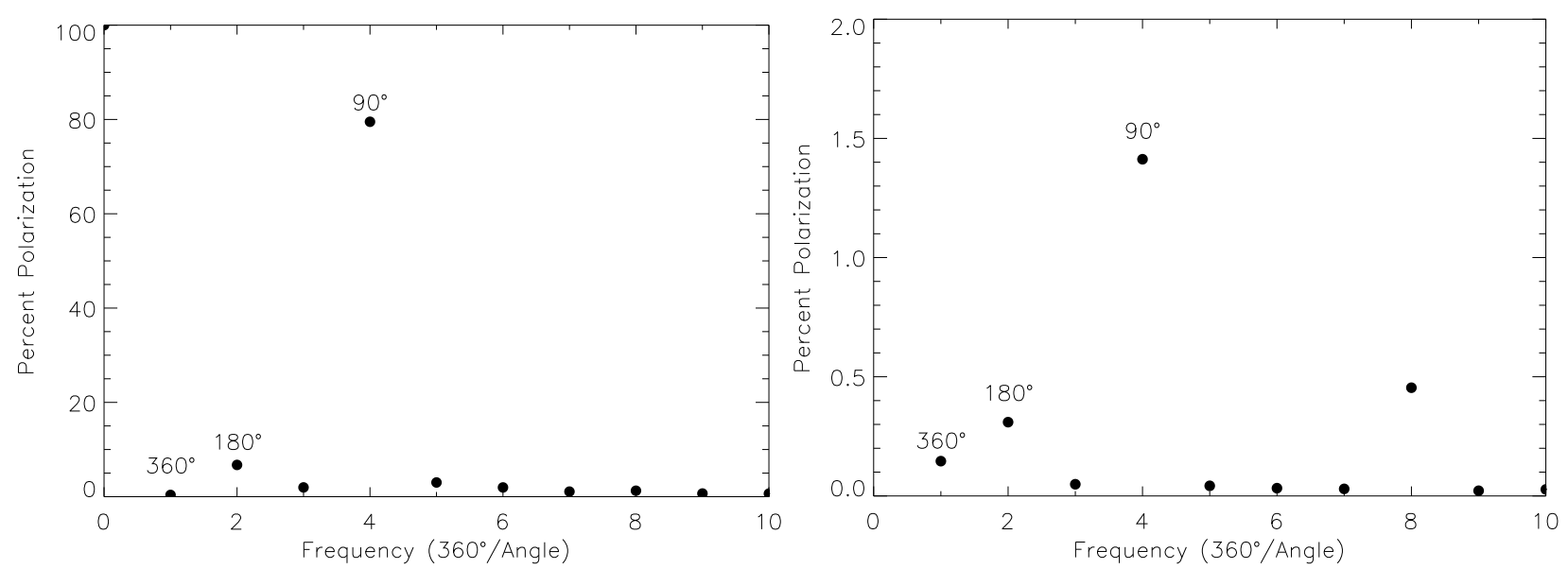

Figure 7. Left: FFT analysis of POLOCAM response as a function of waveplate rotation angle for both $100 \%$ polarized (Left) and unpolarized (Right) source signals. 

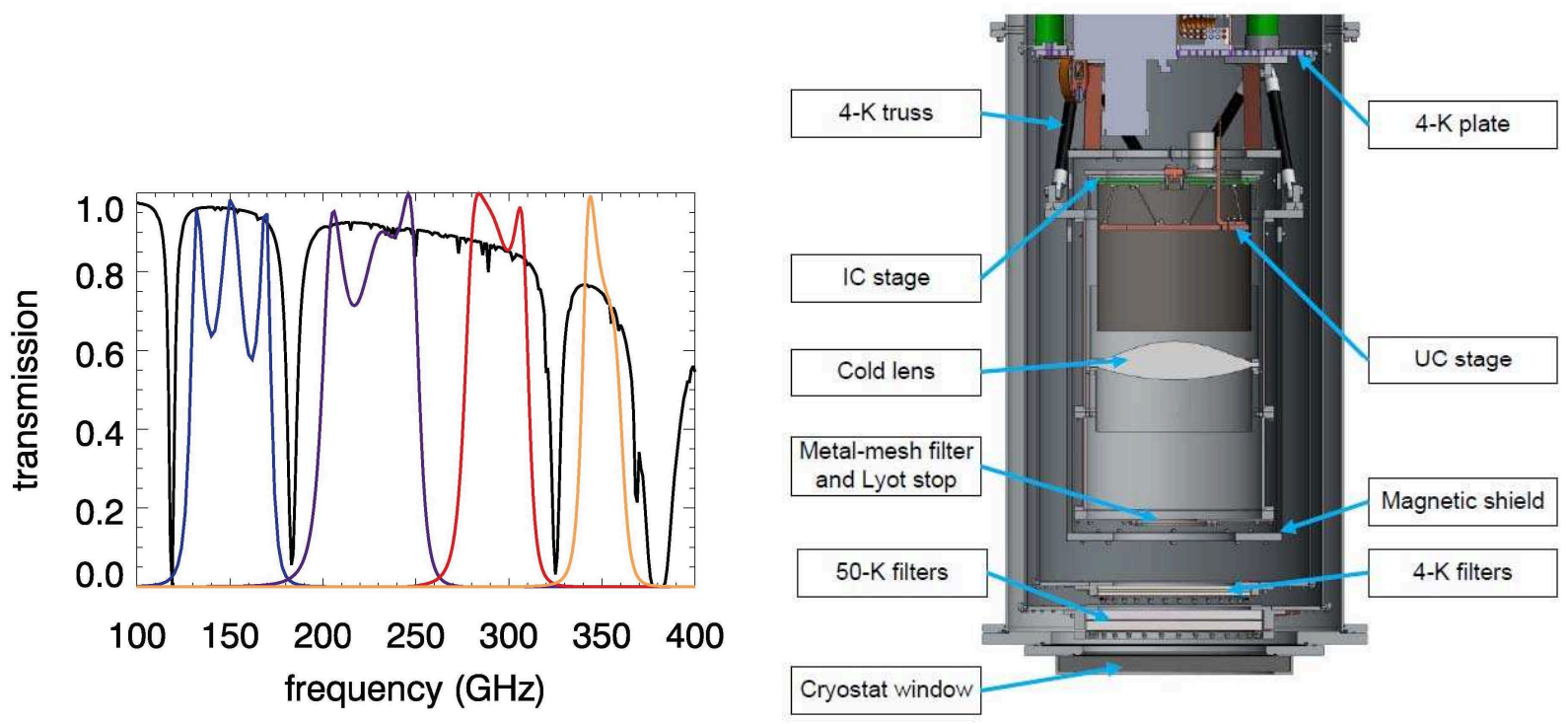

Figure 8. Left: MUSIC filter bands (color) overlaid on the observed atmospheric transmission for 1.5 mm precipitable water vapor at the Caltech Submillimeter Observatory on Mauna Kea. ${ }^{16}$ Right: Cross-section of the cryostat cold hardware and optics, with the key features indicated. ${ }^{20}$

\section{DISCUSSION AND FURTHER CONSIDERATIONS}

We have demonstrated the functionality of the POLOCAM instrument to be a successfully working cryogenic polarimeter prototype for MUSIC-POL. The observed polarization efficiencies and instrumental polarization are in agreement with separate unit-level testing of the test cryostat and waveplate. Performance of the optics, drive mechanism, cryogenic encoder, and warm electronics are as designed.

The existing POLOCAM waveplate is limited in application for the development of MUSIC-POL in two respects due to the concurrent development of MUSIC and POLOCAM. The optimized bandpasses for achromatic behavior of the POLOCAM waveplate $(230,285,345$, and $408 \mathrm{GHz})$ were based on a preliminary design of MUSIC. The actual as-built bandpasses of MUSIC are shown in Figure $8 .{ }^{16}$ The primary changes include the removal of the high frequency $(408 \mathrm{GHz})$ bandpass and the addition of a low-frequency $(150 \mathrm{GHz})$ bandpass. The POLOCAM waveplate performs well in the new bandpass with respect to polarization efficiency, albeit with a higher expected instrumental polarization signal (8\%). In addition, the POLOCAM waveplate diameter $(90 \mathrm{~mm}$ outer diameter with $80 \mathrm{~mm}$ clear aperture) is slightly undersized relative to the as-built MUSIC $4 \mathrm{~K}$ Lyot stop. Physical optics simulations using Zemax indicate that mounting the existing waveplate near the Lyot stop in MUSIC results in minor vignetting for detectors near the edge of the MUSIC focal plane $(10 \%$ at $150 \mathrm{GHz}$ and $5 \%$ at $350 \mathrm{GHz})$ and negligible vignetting for detectors near the center of the focal plane $(0.6 \%$ at $150 \mathrm{GHz}$ and $0.0 \%$ at $350 \mathrm{GHz})$.

\section{ACKNOWLEDGMENTS}

The POLOCAM project is supported by NSF ATI grant 0843916 to Southwest Research Institute. In addition, we acknowledge the University of Colorado for access to laboratory and test facilities and the University of Cardiff for waveplate assembly and testing.

\section{REFERENCES}

[1] Boulares, A. and Cox, D. P., "Galactic hydrostatic equilibrium with magnetic tension and cosmic-ray diffusion," ApJ 365, 544-558 (Dec. 1990).

[2] Shu, F. H., Adams, F. C., and Lizano, S., "Star formation in molecular clouds - Observation and theory," ARAEA 25, 23-81 (1987). 
[3] McKee, C. F., Zweibel, E. G., Goodman, A. A., and Heiles, C., "Magnetic Fields in Star-Forming Regions - Theory," in [Protostars and Planets III], Levy, E. H. and Lunine, J. I., eds., 327 (1993).

[4] Hildebrand, R. H., "Interstellar magnetic fields and infrared-submillimeter spectropolarimetry," in $[A s-$ trophysical Spectropolarimetry], Trujillo-Bueno, J., Moreno-Insertis, F., and Sanchez, F., eds., 265-302, Cambridge University Press, Cambridge (2001).

[5] Shu, F. H., Li, Z.-Y., and Allen, A., "Does Magnetic Levitation or Suspension Define the Masses of Forming Stars?," ApJ 601, 930-951 (Feb. 2004).

[6] Hildebrand, R. H., "Magnetic fields and stardust," QJRAS 29, 327-351 (Sept. 1988).

[7] Lazarian, A., "Magnetic Fields via Polarimetry: Progress of Grain Alignment Theory," J. Quant. Spec. Radiat. Transf. 79, 881 (2003).

[8] Whittet, D. C. B., [Dust in the galactic environment] (1992).

[9] Dotson, J. L., Davidson, J., Dowell, C. D., Schleuning, D. A., and Hildebrand, R. H., "Far-Infrared Polarimetry of Galactic Clouds from the Kuiper Airborne Observatory," ApJS 128, 335-370 (May 2000).

[10] Dotson, J. L., Vaillancourt, J. E., Kirby, L., Dowell, C. D., Hildebrand, R. H., and Davidson, J. A., "350 $\mu \mathrm{m}$ Polarimetry from the Caltech Submillimeter Observatory," ApJS 186, 406-426 (Feb. 2010).

[11] Matthews, B. C., McPhee, C. A., Fissel, L. M., and Curran, R. L., "The Legacy of SCUPOL: $850 \mu \mathrm{m}$ Imaging Polarimetry from 1997 to 2005," ApJS 182, 143-204 (May 2009).

[12] Kogut, A., Dunkley, J., Bennett, C. L., Doré, O., Gold, B., Halpern, M., Hinshaw, G., Jarosik, N., Komatsu, E., Nolta, M. R., Odegard, N., Page, L., Spergel, D. N., Tucker, G. S., Weiland, J. L., Wollack, E., and Wright, E. L., "Three-Year Wilkinson Microwave Anisotropy Probe (WMAP) Observations: Foreground Polarization," ApJ 665, 355-362 (Aug. 2007).

[13] Li, H., Griffin, G. S., Krejny, M., Novak, G., Loewenstein, R. F., Newcomb, M. G., Calisse, P. G., and Chuss, D. T., "Results of SPARO 2003: Mapping Magnetic Fields in Giant Molecular Clouds," ApJ 648, 340-354 (Sept. 2006).

[14] Clavel, J. and Tauber, J. A., "The Planck Mission," in [EAS Publications Series], Gurvits, L. I., Frey, S., and Rawlings, S., eds., EAS Publications Series 15, 395-403 (2005).

[15] Planck Collaboration, Abergel, A., Ade, P. A. R., Aghanim, N., Arnaud, M., Ashdown, M., Aumont, J., Baccigalupi, C., Balbi, A., Banday, A. J., Barreiro, R. B., Bartlett, J. G., Battaner, E., Benabed, K., Benoît, A., Bernard, J.-P., Bersanelli, M., Bhatia, R., Bock, J. J., Bonaldi, A., Bond, J. R., Borrill, J., Bouchet, F. R., Boulanger, F., Bucher, M., Burigana, C., Cabella, P., Cardoso, J.-F., Catalano, A., Cayón, L., Challinor, A., Chamballu, A., Chiang, L.-Y., Chiang, C., Christensen, P. R., Clements, D. L., Colombi, S., Couchot, F., Coulais, A., Crill, B. P., Cuttaia, F., Danese, L., Davies, R. D., Davis, R. J., de Bernardis, P., de Gasperis, G., de Rosa, A., de Zotti, G., Delabrouille, J., Delouis, J.-M., Désert, F.-X., Dickinson, C., Dobashi, K., Donzelli, S., Doré, O., Dörl, U., Douspis, M., Dupac, X., Efstathiou, G., Enßlin, T. A., Eriksen, H. K., Finelli, F., Forni, O., Frailis, M., Franceschi, E., Galeotta, S., Ganga, K., Giard, M., Giardino, G., Giraud-Héraud, Y., González-Nuevo, J., Górski, K. M., Gratton, S., Gregorio, A., Gruppuso, A., Guillet, V., Hansen, F. K., Harrison, D., Henrot-Versillé, S., Herranz, D., Hildebrandt, S. R., Hivon, E., Hobson, M., Holmes, W. A., Hovest, W., Hoyland, R. J., Huffenberger, K. M., Jaffe, A. H., Jones, A., Jones, W. C., Juvela, M., Keihänen, E., Keskitalo, R., Kisner, T. S., Kneissl, R., Knox, L., Kurki-Suonio, H., Lagache, G., Lamarre, J.-M., Lasenby, A., Laureijs, R. J., Lawrence, C. R., Leach, S., Leonardi, R., Leroy, C., LindenVørnle, M., López-Caniego, M., Lubin, P. M., Macías-Pérez, J. F., MacTavish, C. J., Maffei, B., Mandolesi, N., Mann, R., Maris, M., Marshall, D. J., Martin, P., Martínez-González, E., Masi, S., Matarrese, S., Matthai, F., Mazzotta, P., McGehee, P., Meinhold, P. R., Melchiorri, A., Mendes, L., Mennella, A., Mitra, S., Miville-Deschênes, M.-A., Moneti, A., Montier, L., Morgante, G., Mortlock, D., Munshi, D., Murphy, A., Naselsky, P., Natoli, P., Netterfield, C. B., Nørgaard-Nielsen, H. U., Noviello, F., Novikov, D., Novikov, I., Osborne, S., Pajot, F., Paladini, R., Pasian, F., Patanchon, G., Perdereau, O., Perotto, L., Perrotta, F., Piacentini, F., Piat, M., Plaszczynski, S., Pointecouteau, E., Polenta, G., Ponthieu, N., Poutanen, T., Prézeau, G., Prunet, S., Puget, J.-L., Reach, W. T., Rebolo, R., Reinecke, M., Renault, C., Ricciardi, S., Riller, T., Ristorcelli, I., Rocha, G., Rosset, C., Rubiño-Martín, J. A., Rusholme, B., Sandri, M., Santos, D., Savini, G., Scott, D., Seiffert, M. D., Shellard, P., Smoot, G. F., Starck, J.-L., Stivoli, F., Stolyarov, V., Sudiwala, R., Sygnet, J.-F., Tauber, J. A., Terenzi, L., Toffolatti, L., Tomasi, M., Torre, J.-P., Tristram, M., Tuovinen, J., Umana, G., Valenziano, L., Verstraete, L., Vielva, P., Villa, F., Vittorio, N., Wade, L. A., 
Wandelt, B. D., Yvon, D., Zacchei, A., and Zonca, A., "Planck early results. XXV. Thermal dust in nearby molecular clouds," A $\mathscr{S} A$ 536, A25 (Dec. 2011).

[16] Maloney, P. R., Czakon, N. G., Day, P. K., Downes, T. P., Duan, R., Gao, J., Glenn, J., Golwala, S. R., Hollister, M. I., Leduc, H. G., Mazin, B. A., McHugh, S. G., Noroozian, O., Nguyen, H. T., Sayers, J., Schlaerth, J. A., Siegel, S., Vaillancourt, J. E., Vayonakis, A., Wilson, P., and Zmuidzinas, J., "MUSIC for sub/millimeter astrophysics," in [Society of Photo-Optical Instrumentation Engineers (SPIE) Conference Series], Society of Photo-Optical Instrumentation Engineers (SPIE) Conference Series $\mathbf{7 7 4 1}$ (July 2010).

[17] Mazin, B. A., Microwave kinetic inductance detectors, PhD thesis, California Institute of Technology, California, USA (2005).

[18] Glenn, J., Day, P. K., Ferry, M., Gao, J., Golwala, S. R., Kumar, S., LeDuc, H. G., Maloney, P. R., Mazin, B. A., Nguyen, H., Noroozian, O., Sayers, J., Schlaerth, J., Vaillancourt, J. E., Vayokanis, A., and Zmuidzinas, J., "A microwave kinetic inductance camera for sub/millimeter astrophysics," in [Society of Photo-Optical Instrumentation Engineers (SPIE) Conference Series], Society of Photo-Optical Instrumentation Engineers (SPIE) Conference Series 7020 (Aug. 2008).

[19] Zhang, J., Ade, P. A. R., Mauskopf, P., Moncelsi, L., Savini, G., and Whitehouse, N., "New artificial dielectric metamaterial and its application as a terahertz antireflection coating," Appl. Opt. 48, 6635-6642 (Dec. 2009).

[20] Hollister, M. I., Czakon, N. G., Day, P. K., Downes, T. P., Duan, R., Gao, J., Glenn, J., Golwala, S. R., Leduc, H. G., Maloney, P. R., Mazin, B. A., Nguyen, H. T., Noroozian, O., Sayers, J., Schlaerth, J., Siegel, S., Vaillancourt, J. E., Vayonakis, A., Wilson, P., and Zmuidzinas, J., "The cryomechanical design of MUSIC: a novel imaging instrument for millimeter-wave astrophysics at the Caltech Submillimeter Observatory," in [Society of Photo-Optical Instrumentation Engineers (SPIE) Conference Series], Society of Photo-Optical Instrumentation Engineers (SPIE) Conference Series 7741 (July 2010). 